\title{
Abundance analysis of late B stars ${ }^{\star}$ \\ Evidence for diffusion and against weak stellar winds
}

\begin{abstract}
M. Hempel ${ }^{1}$ and H. Holweger ${ }^{2}$
1 Hamburger Sternwarte, Gojenbergsweg 112, 21029 Hamburg, Germany

2 Institut für Theoretische Physik und Astrophysik, Universität Kiel, 24098 Kiel, Germany

Received 14 January 2003 / Accepted 2 June 2003

Abstract. Based on high S/N spectra obtained at La Silla, Chile, and the Special Astrophysical Observatory, Russia, the abundances of $\mathrm{He}, \mathrm{C}, \mathrm{O}, \mathrm{Ne}, \mathrm{Mg}, \mathrm{Si}, \mathrm{Ca}, \mathrm{Fe}, \mathrm{Sr}$, and $\mathrm{Ba}$ in 27 optically bright B5-B9 main-sequence stars were determined. NLTE effects were taken into account. A variety of abundance patterns is present in late B stars. Accurate surface abundances of the diffusion indicators $\mathrm{O}, \mathrm{Mg}, \mathrm{Ca}, \mathrm{Sr}$ and Ba suggest that element stratification due to diffusion is common in the program stars. Models of stellar atmospheres which include meridional mixing can explain the observed anomalies. Although the program stars represent only a volume-limited sample of the solar neighbourhood this result is important for the cosmochemical evolution of the Galaxy: the surface abundances of the stars investigated do not necessarily reflect the chemical composition of the interstellar cloud they originated from. Furthermore, five program stars show narrow absorption lines in Ca II K which can be attributed to circumstellar gas. Neon serves as a trace element for the occurrence of weak stellar winds. Neon overabundances of some stars derived under the assumption of LTE suggest that such winds have been detected. In sharp contrast, the more realistic treatment of NLTE leads to solar neon abundances and thus reveals that weak stellar winds are absent in the program stars.
\end{abstract}

Key words. stars: abundances - stars: atmospheres - stars: chemically peculiar - stars: winds, outflows

\section{Introduction}

In stars of the upper main-sequence the increasing effective temperatures cause the ionisation zones of hydrogen and helium to be shifted more and more towards the stellar surface. As a result the outer convection zones of these stars become thinner and less turbulent. But this absence of turbulent convection does not necessarily lead to ideal, static atmospheres. Some A and B stars show spectroscopic signatures of microscopic and macroscopic transport processes. The diagnostics of these processes yield valuable information about the behaviour of an element under the influence of gravitation and radiation. Even in nonmagnetic stars this leads to a variety of abundance patterns.

On the cooler end of the upper main-sequence the well known Fm-Am stars are not exceptional (Wolff 1983). Michaud (1970) invoked diffusion processes as an explanation for those abundance anomalies. Since then various studies on both theoretical and observational aspects of abundance anomalies have been carried out (see, e.g., Gonzalez et al. 1995; Alecian 1996). The basic idea is that the competition of gravitative settling and

Send offprint requests to: $\mathrm{M}$. Hempel,

e-mail: mhempel@hs .uni-hamburg.de

* Based on observations collected at the European Southern Observatory, La Silla, Chile and at the Special Astrophysical Observatory, Nizhnij Arkhyz, Russia. radiative levitation leads to element separation via diffusion that occurs directly below the convection zone. Depending on its absorption cross-section a certain element in this reservoir will either be enriched or depleted. With the atomic data from the Opacity Project (Seaton et al. 1992) former shortcomings of theoretical calculations could be improved (Gonzales et al. 1995). Abundance determinations of A stars classified as "normal" have shown a variety of abundance anomalies (Holweger et al. 1986; Gigas 1986, 1988; Lemke 1989, 1990). In normal A stars diffusion leads to deficiencies of $\mathrm{Ca}$ as well as overabundances of strontium and barium.

In the same temperature regime the $\lambda$-Bootis-phenomenon may occur in pre-main-sequence stars. The characteristic metal underabundance of these young stars is attributed to a different mechanism of element separation: the accretion of metaldeficient gas from the circumstellar environment after gas-dustseparation (Venn \& Lambert 1990). As a result the volatile elements carbon, nitrogen, and oxygen have nearly solar abundances while heavier elements with higher condensation temperatures are locked up in the dust grains and therefore are deficient in the stellar atmospheres (Stürenburg 1993; Paunzen et al. 1999). The $\lambda$-Bootis-phenomenon is believed to occur in pre-main-sequence stars at the end of their accretion phase (Holweger \& Rentzsch-Holm 1995). Depending on the accretion rate the interplay of accretion and diffusion can lead to 
over- or underabundances of certain elements. In fast rotators, meridional circulation can reduce these abundance anomalies (Turcotte \& Charbonneau 1993). The comparatively short phase of pre-main-sequence evolution is consistent with the paucity of the $\lambda$-Bootis stars. Interestingly, the standard star Vega is believed to be a member of this group.

On the hot end of the main-sequence, radiative processes are dominant and lead to massive radiatively-driven stellar winds in O stars and early-type B stars (Kudritzki \& Hummer 1990). These stellar winds may lead to spectroscopic signatures of substantial mass loss (Kilian 1992). Furthermore, in fast rotators products of nuclear fusion can emerge to the stellar surface. In spite of their rarity, these stars play an important role in the enrichment of the interstellar medium with elements like carbon, nitrogen, or oxygen.

The transition between the diffusion-accretion dominated atmospheres and the wind-driven atmospheres lies in the region of the late B stars which are subject of this study. Theoretical approaches suggest that with increasing effective temperatures from the regime of the A stars, selective stellar winds set in (Babel 1995) which blow away metals with large absorption cross sections due to radiation pressure. One can expect that such a "metallic" wind changes the composition of the stellar surface, and may even contribute to the metallicity of the interstellar medium. Furthermore, Landstreet et al. (1998) suggests certain elements, like neon, are tracers for the detection of weak stellar winds: overabundances of these elements provide valuable evidence for the presence of weak stellar winds in the order of $10^{-14} M_{\odot} / y r$. Up to now, these weak stellar winds have not been detected spectroscopically. Their detection would be of great importance for diffusion theories as they often have to be invoked to explain mild discrepancies between observations and models (see, e.g., Alecian 1996).

To date the previously described transition region in the range of the late $B$ stars has been investigated only very sparsely, and literature on the processes of element separation is scarce. The status of research is documented in the studies of Adelman \& Philip (1996), Smith \& Dworetsky (1993), and Smith (1993, 1996). Adelman \& Philip (1996) state that "The general pattern of subsolar abundances among $B$ stars [...] is contrary to what is expected for stars which are much younger than the Sun". In some of the 10 investigated northern B stars, abundance anomalies are found, but a diagnosis is carried out only in view of the chemical evolution of the Galaxy. In addition, the data are based largely on older photographic analysis, including low-dispersion, photographic spectra. Smith and Dworetsky analyse IUE-spectra of 8 normal B 5-B 9.5 stars of luminosity classes V and IV. Their result, "Approximately solar abundances of these elements are obtained for the normal stars", is obviously contradictory to the findings of Adelman \& Philip (1996). This suggests that in the crowded UV line spectrum of these stars, only large abundance anomalies can be traced. In all the mentioned papers deviations from LTE are neglected. Some of the stars investigated in this study have been analysed by Wohler (1996) and van Thiel (1997) assuming LTE. Their findings suggest star-to-star variations of carbon, magnesium, calcium, and iron, but no definite indications for diffusion could be found. Depending on the ionisation stage and the line strength, deviations from LTE can become significant, and neglecting NLTE effects - as usual in the case of A and late B stars - can conceal abundance patterns and make correlations insignificant.

By investigating the abundances of key elements for element separation processes, the present study aims at a coherent picture of the chemical composition of 27 late B stars based on optical spectra. Deviations from LTE are taken into account, and the results are discussed in view of diffusion processes and weak stellar winds.

\section{Observations}

We have studied 27 B5-B9 stars (see Table 1) taken from the Bright Star Catalogue (Hoffleit \& Warren 1991, BSC) and classified as "normal", i.e. they neither have peculiar spectra nor emission lines. The sample consists of 20 southern and 7 northern hemisphere stars. The spectra of 20 stars have been collected by A. Kaufer in February and March 1995 with the ESO-50-cm telescope equipped with the HEROS spectrograph. The spectra have a resolution of $R=20000$. Data reduction and wavelength calibration was carried out by A. Kaufer using MIDAS.

The spectra of the 7 other stars have been obtained by G.A. Galazutdinov and F. A. Musaev in March 1999 with the SAO 1-m-telescope using the echelle spectrograph. The wavelength range covers $3500 \AA$ to $10000 \AA$ with a resolution of $R=45000$. Data reduction, as well as wavelength calibration, was carried out by the observers using their echelle software package. The last column of Table 1 indicates the site of observation. The spectra of both datasets have a $\mathrm{S} / \mathrm{N}$ of about 100 .

\section{Model-atmosphere analysis}

The basic stellar parameters $T_{\text {eff }}$ and $\log g$ (see Table 1) have been obtained via Strömgren photometry (Hauck \& Mermillod 1990) using the calibration of Napiwotzki et al. (1993). The ATLAS9 code (Kurucz 1993) was used to calculate $T(\tau)$ relations assuming solar metallicity. The temperature structure serves as an input to our LTE/NLTE system. Pressures and particle concentrations were derived from the $T(\tau)$ relations using our ATMOS code. The spectrum synthesis was carried out with our line formation code LINFOR (Lemke 1991). Lemke (1989) found a microturbulence of $\xi \approx 2 \mathrm{~km} \mathrm{~s}^{-1}$ in his analysis of A stars, and the work of Fitzpatrick \& Massa (1999) on the physical properties of B stars reveals microturbulent velocities between $0 \mathrm{~km} \mathrm{~s}^{-1}$ and $1.9 \mathrm{~km} \mathrm{~s}^{-1}$ for six out of seven objects having similar $T_{\text {eff }}$ and $\log g$ values to those of our program stars. In accordance with the findings of Krege (1995) we adopted a microturbulence of $\xi=1 \mathrm{~km} \mathrm{~s}^{-1}$. We note that our NLTE abundance analysis of Si II and Fe II lines of different strengths revealed consistent abundances of weak and strong lines. This suggests that $\xi=1 \mathrm{~km} \mathrm{~s}^{-1}$ is a good estimate of this poorly defined parameter. The VALD database (Piskunov et al. 1995) was used to select lines for abundance analysis and to identify blend lines. The $\log g f$ values were also taken from the VALD compilation, except for carbon and oxygen for which we used the more recent data of Wiese et al. (1996). 
Table 1. Parameters of the program stars. The data were taken from the Bright Star Catalogue (Hoffleit \& Warren 1991). Distances have been calculated using HIPPARCOS parallaxes (ESA 1997). The last column indicates whether a star was observed from ESO (E) or SAO (S).

\begin{tabular}{|c|c|c|c|c|c|c|c|c|c|c|c|}
\hline \multirow[t]{2}{*}{ HR } & \multirow[t]{2}{*}{ Name } & \multirow[t]{2}{*}{ HD } & \multirow[t]{2}{*}{$m_{v}$} & \multirow{2}{*}{$\begin{array}{l}\text { Sp. } \\
\text { Type }\end{array}$} & \multirow{2}{*}{$\begin{array}{l}T_{\text {eff }} \\
(\mathrm{K})\end{array}$} & \multirow{2}{*}{$\begin{array}{c}\log g \\
\left(\mathrm{~cm} \mathrm{~s}^{-2}\right) \\
\end{array}$} & \multirow{2}{*}{$\begin{array}{r}v \sin i \\
\left(\mathrm{~km} \mathrm{~s}^{-1}\right)\end{array}$} & \multirow{2}{*}{$\begin{array}{c}d \\
(\mathrm{pc})\end{array}$} & RA & \multirow{2}{*}{$\begin{array}{l}\text { DEC } \\
, 1,\end{array}$} & \multirow[t]{2}{*}{ Obs } \\
\hline & & & & & & & & & $\mathrm{h} \mathrm{m} \mathrm{s}$ & & \\
\hline 806 & $\epsilon$ Hyi & 16978 & 4.11 & B9 V & 10910 & 4.29 & $100 \pm 2$ & 47.01 & 023935 & -681601 & $\mathrm{E}$ \\
\hline 1070 & 17 Eri & 21790 & 4.73 & B9 V & 11540 & 3.61 & $86 \pm 2$ & 116.69 & 033037 & -050431 & E \\
\hline 1092 & & 22252 & 5.83 & B8 V & 11860 & 3.27 & $260 \pm 15$ & 261.10 & 033052 & -662923 & $\mathrm{E}$ \\
\hline 1214 & & 24626 & 5.11 & B6 V & 14300 & 4.12 & $29 \pm 2$ & 108.81 & 035339 & -344356 & $\mathrm{E}$ \\
\hline 1339 & $53 \mathrm{Tau}$ & 27295 & 5.35 & B9 IV & 12000 & 4.19 & $5 \pm 2$ & 81.96 & 041926 & +210832 & $\mathrm{~S}$ \\
\hline 1582 & 62 Eri & 31512 & 5.51 & B6 V & 15190 & 3.87 & $89 \pm 4$ & 226.76 & 045624 & - 051017 & $\mathrm{E}$ \\
\hline 1723 & & 34310 & 5.07 & B9 V & 11180 & 4.12 & $158 \pm 2$ & 83.47 & 051524 & -265636 & $\mathrm{E}$ \\
\hline 1728 & 17 Aur & 34364 & 6.14 & B9.5 V & 11010 & 4.34 & $30 \pm 4$ & 121.95 & 051819 & +334602 & S \\
\hline 1973 & & 38170 & 5.29 & B9.5 V & 10250 & 3.69 & $60 \pm 4$ & 111.24 & 054215 & -344004 & E \\
\hline 2056 & $\lambda \mathrm{Col}$ & 39764 & 4.87 & B5 V & 15370 & 4.22 & $101 \pm 3$ & 104.71 & 055307 & -334805 & E \\
\hline 2948 & & 61555 & 4.50 & B6 V & 15460 & 4.20 & $50 \pm 5$ & 139.28 & 073849 & -264806 & E \\
\hline 3158 & & 66552 & 6.15 & B9 V & 10450 & 4.09 & $75 \pm 4$ & 91.57 & 080445 & +185032 & S \\
\hline 3439 & & 74067 & 5.20 & B9 V & 10880 & 4.14 & $36 \pm 3$ & 85.98 & 084019 & -405115 & E \\
\hline 3717 & & 80781 & 6.28 & B5 V & 14980 & 3.22 & $30 \pm 3$ & 1052.63 & 091933 & -551112 & $\mathrm{E}$ \\
\hline 4116 & $\delta$ Sex & 90882 & 5.21 & B9.5 V & 10360 & 3.84 & $140 \pm 3$ & 91.99 & 102929 & -024421 & $S$ \\
\hline 4119 & $\beta$ Sex & 90994 & 5.09 & B6 V & 14570 & 4.21 & $85 \pm 4$ & 105.71 & 103018 & -003813 & $\mathrm{E}$ \\
\hline 4943 & $14 \mathrm{CVn}$ & 113797 & 5.25 & B9 V & 11260 & 4.06 & $130 \pm 3$ & 86.58 & 130545 & +354756 & S \\
\hline 5501 & $108 \mathrm{Vir}$ & 129956 & 5.69 & B9.5 V & 10250 & 3.75 & $84 \pm 4$ & 188.32 & 144530 & +004302 & E \\
\hline 5685 & $\beta \mathrm{Lib}$ & 135742 & 2.61 & B8 V & 12040 & 3.26 & $320 \pm 20$ & 49.06 & 151700 & -092259 & S \\
\hline 5994 & $\iota^{2}$ Nor & 144480 & 5.57 & B9.5 V & 10580 & 4.29 & $87 \pm 3$ & 83.19 & 160919 & -575604 & $\mathrm{E}$ \\
\hline 6628 & & 161840 & 4.83 & B8 V & 11990 & 3.38 & $38 \pm 2$ & 186.22 & 174911 & -314212 & $\mathrm{E}$ \\
\hline 6633 & & 161941 & 6.22 & B9.5 V & 9960 & 3.26 & $36 \pm 3$ & 757.58 & 174820 & +034815 & $\mathrm{E}$ \\
\hline 6647 & & 162374 & 5.90 & B6 V & 16900 & 3.72 & $38 \pm 2$ & 254.45 & 175214 & -344757 & $\mathrm{E}$ \\
\hline 6668 & & 162817 & 5.96 & B9.5 V & 9190 & 3.15 & $76 \pm 4$ & 258.40 & 175427 & - 342759 & E \\
\hline 6878 & & 169009 & 6.33 & B9.5 V & 10440 & 3.95 & $47 \pm 5$ & 105.15 & 182302 & -101307 & E \\
\hline 7337 & $\beta^{1} \mathrm{Sgr}$ & 181454 & 4.01 & B9 V & 11960 & 3.83 & $77 \pm 2$ & 116.01 & 192238 & -442732 & E \\
\hline 8781 & $\alpha$ Peg & 218045 & 2.49 & B9 V & 9810 & 3.51 & $130 \pm 2$ & 42.80 & 230446 & +151219 & $\mathrm{~S}$ \\
\hline
\end{tabular}

B stars are known to have very large rotational velocities, up to $v \sin i \approx 350 \mathrm{~km} \mathrm{~s}^{-1}$, which lead to extremely broadened line spectra. This causes difficulties for a reliable spectrum synthesis. Therefore we have selected the 20 southern hemisphere objects with $v \sin i<100 \mathrm{~km} \mathrm{~s}^{-1}$. We have dropped this restriction for the 7 northern stars. The reason is the detection of narrow absorption features in the Ca II $\mathrm{K}$ line and will be discussed in Sect. 6. Nevertheless the rotational velocities (listed in Table 1) we derived via spectrum synthesis reveal large errors of the values given in the BSC.

Based on the assumptions that $\Delta T_{\text {eff }}=200 K, \Delta \log g=$ $0.2 \mathrm{dex}$, and $\Delta \xi=1 \mathrm{~km} \mathrm{~s}^{-1}$ we carried out calculations in order to estimate the errors of our analysis. Both effective temperature and $\log g$ variations lead to maximum abundance uncertainties of $\pm 0.1 \mathrm{dex}$, and variation of $\xi$ yielded abundance changes below \pm 0.05 dex. Thus we found that the typical error of our abundances is between 0.2 and 0.3 dex.

\section{LTE-abundances}

Apart from iron, solar abundances are from Anders and Grevesse (1989); for iron the revised value of 7.51 Holweger et al. (1995) was used. Lines of ten elements, namely helium, carbon, oxygen, neon, magnesium, silicon, calcium, iron, strontium, and barium, were selected for abundance analysis. The lines are compiled in Table 2. Additionally, we included all relevant blend lines.
A closer look at the LTE results in Tables 5-7 reveals that some stars show conspicuous deviations from solar values clearly above the error limit of $0.3 \mathrm{dex}$. Oxygen shows pronounced overabundances in most stars, and neon is overabundant in some objects by nearly 0.6 dex. Studies on oxygen (Baschek et al. 1977; Takeda 1992; Paunzen et al. 1999) and neon abundances (Dworetsky \& Budaj 2000) reveal that these elements are expected to show large non-LTE effects. The values for magnesium scatter largely between -1.3 dex and 0.31 dex with most abundances being subsolar. Nevertheless, some stars have definitely non-solar magnesium abundances. Silicon and iron show scatter around the normal, solar abundances. Calcium has obvious underabundances in some objects. Lastly, the heavy elements strontium and barium are overabundant in most cases.

To sum this up one can state that clear compositional differences have been found in the program stars. The question arises whether these abundance patterns are real or due to the inadequate assumption of LTE. To investigate this the NLTE calculations outlined in the next section were carried out.

\section{NLTE corrections}

NLTE corrections were carried out using the Kiel NLTE-code (Steenbock \& Holweger 1984) for C, O, Ne, Mg, Si, Ca, Fe, $\mathrm{Sr}$, and $\mathrm{Ba}$. In the following some details concerning the model atoms are provided. 

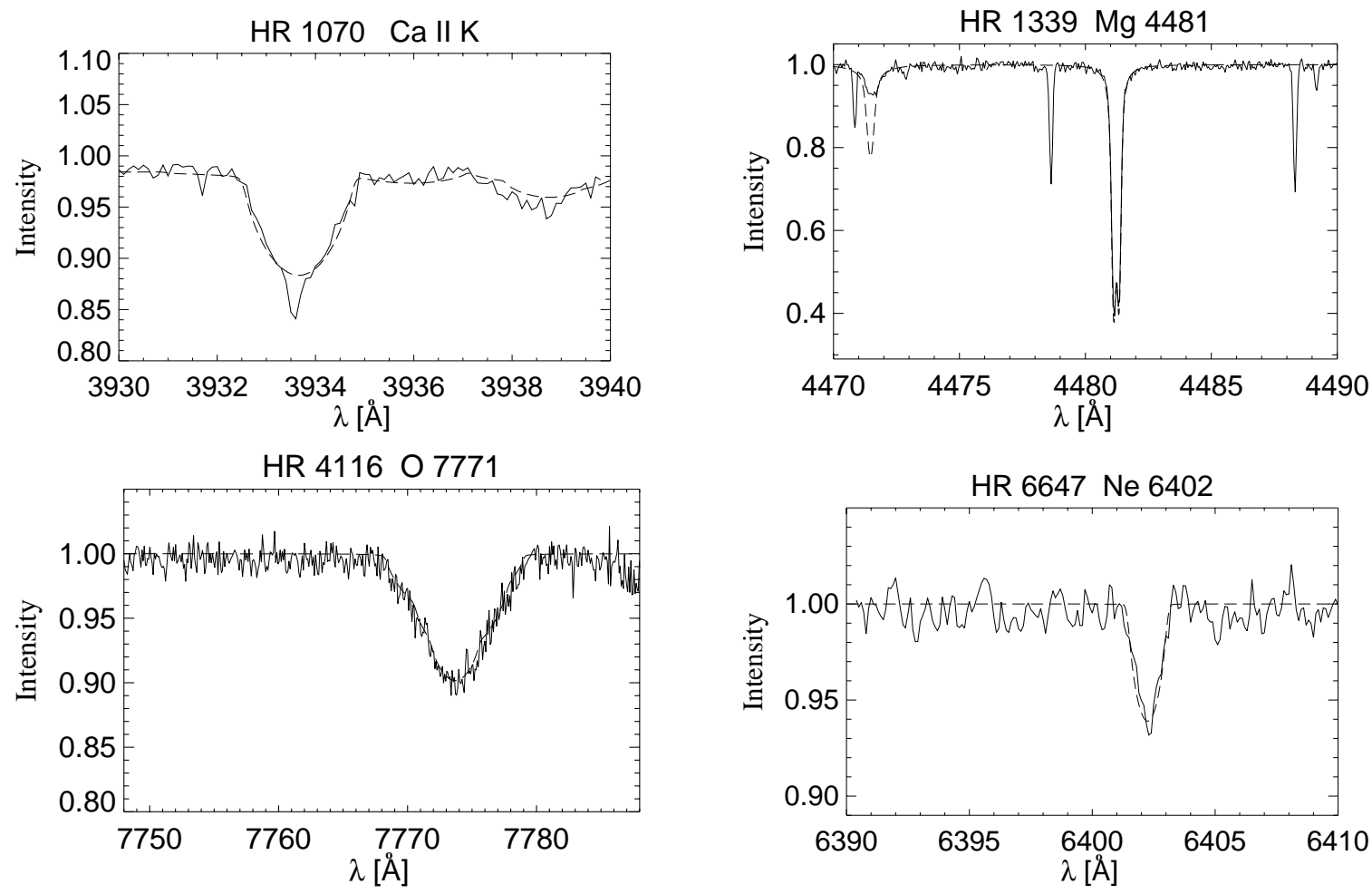

Fig. 1. Some typical observed (solid lines) and synthetic spectra (dashed lines). Note the narrow absorption feature in the core of the Ca II K line of HR 1070 (see Sect. 6 for details).

\subsection{Carbon}

The carbon model atom by I. Kamp (Rentzsch-Holm 1996) contains 83 CI levels, 15 levels of C II and 79 line transitions. It is an improved version of an older $\mathrm{CI} / \mathrm{II}$ atom with a total of 88 levels and 66 transitions (Stürenburg \& Holweger 1990). A detailed description of the atom can be found in Rentzsch-Holm (1996). Although the NLTE corrections are not negligible, they are not very pronounced for most of the program stars which is in accordance with the findings of Rentzsch-Holm (1996). In fact, only for four stars of the twelve objects where carbon lines have been analyzed are the corrections above 0.2 dex. No temperature dependency can be found for the sample stars. As outlined by Stürenburg \& Holweger (1990) and Rentzsch-Holm (1996) NLTE corrections of strong carbon lines depend on the equivalent width. This effect is only important for lines with $W_{\lambda}>100 \mathrm{~m} \AA$. Because the lines of the program stars are weaker this is not observed here. The NLTE abundances of most stars investigated here scatter around the solar value.

\subsection{Oxygen}

The OI 7771-5 triplet investigated in this study shows large non-LTE effects as outlined by Baschek et al. (1977) and Takeda (1992). Furthermore, from previous work on A stars (Paunzen et al. 1999) non-LTE corrections of up to -0.7 dex can be expected. An O I model atom containing 15 energy levels and 17 transitions has been developed by I. Kamp \& M. Hempel for the analysis of $\lambda$ Bootis stars
(Paunzen et al. 1999). This model atom is not appropriate for the application to B stars having sufficiently higher temperatures. Therefore an improved version was developed which is based on the old one described in Paunzen et al. (1999). It contains 29 energy levels plus the continuum of O II and 71 line transitions. The energy levels have been obtained from the Atomic Spectra Database of NIST ${ }^{1}$. All energy levels up to $5 \mathrm{f}$ at $13.07 \mathrm{eV}$ were implemented and are listed in Table 3. Higher levels will only have negligible effects. When available, energy resolved electron collisional crosssections were adopted. The values for the $2 \mathrm{p}^{3} \mathrm{P}-2 \mathrm{p}^{1} \mathrm{D}$ and $2 p^{3} P-2 p^{1} S$ transitions were adopted from Tayal (1992), for $2 \mathrm{p}^{3} \mathrm{P}-3 \mathrm{~s}^{3}$ So, $2 \mathrm{p}^{3} \mathrm{P}-3 \mathrm{~s}^{3}$ Do, and $2 \mathrm{p}^{3} \mathrm{P}-3 \mathrm{~d}^{3}$ Do the data from Wang \& Mc Conkey (1992) were used. Tayal \& Henry (1989) provide values for $2 \mathrm{p}^{3} \mathrm{P}-3 \mathrm{~s}^{5} \mathrm{So}, 2 \mathrm{p}^{3} \mathrm{P}-3 \mathrm{p}^{5} \mathrm{P}, 2 \mathrm{p}^{3} \mathrm{P}-4 \mathrm{~s}^{3}$ So, and $2 \mathrm{p}^{3} \mathrm{P}-4 \mathrm{p}^{3} \mathrm{P}$. Allowed electron collisional cross-sections missing were calculated according to Van Regemorter (1962). Concerning the electron cross-sections for optically forbidden transitions the formula given by Allen (1973)

$\sigma_{\max }=\frac{\Omega}{g_{l}} \frac{E_{H}}{\Delta E} \pi a_{0}^{2}$

was applied. In (1) $\Omega$ denotes the collision strength, assuming $\Omega=1, g_{l}$ is the statistical weight of the lower level, $E_{H}$ is the energy difference between lower and upper level, and $a_{0}^{2}$ is the Bohr radius. Collisions with neutral hydrogen atoms were calculated following Steenbock \& Holweger (1984). Because hydrogen is primarily ionised, electron collisions are dominant and collisions with neutral hydrogen are not relevant for

${ }^{1}$ http://physics.nist.gov/PhysRefData/contents.html 
Table 2. This table comprises all lines used for the abundance analysis. In Cols. 3 and 4 we compiled the contributions of blend lines and additional comments, respectively.

\begin{tabular}{|c|c|c|c|}
\hline Element/Ion & Wavelengths & Blends & Comments \\
\hline \multirow[t]{3}{*}{ Helium } & He I 5876 & weak Fe II & blend of $5 \mathrm{He} \mathrm{I} \mathrm{lines}$ \\
\hline & He I 6678 & weak Fe II/Si II $\gtrsim 15000 \mathrm{~K}$ & He I 6 times stronger than blends \\
\hline & He I 7065 & none & useful $\gtrsim 12000 \mathrm{~K}$ \\
\hline \multirow[t]{4}{*}{ Carbon } & C II 3918/3920 & $\mathrm{H}_{\zeta}(3889 \AA), \mathrm{Ca}$ II K $(3934 \AA)$ & blends have well-known $g f$ values, clearly traceable \\
\hline & & weak Fe II/Ti II/S II & blends become weaker with rising $T_{\text {eff }}$ \\
\hline & C II 4267 & weak $\mathrm{Fe} \mathrm{I/Fe} \mathrm{II/S} \mathrm{II}$ & blend of $3 \mathrm{C}$ II lines, C II becomes stronger with rising $T_{\mathrm{eff}}$ \\
\hline & C II 6578 & weak Fe II & C II becomes stronger with with rising $T_{\text {eff }}$ \\
\hline Oxygen & O I 7771 & weak Fe II on red wing & O I triplet, suitable for the determination of $v \sin i$ \\
\hline \multirow[t]{2}{*}{ Neon } & Ne I 6402 & none & tracer for weak stellar winds, useful $\gtrsim 11500 \mathrm{~K}$ \\
\hline & Ne I 6506 & none & tracer for weak stellar winds, useful $\gtrsim 11500 \mathrm{~K}$ \\
\hline Magnesium & Mg II 4481 & $\begin{array}{l}\text { weak Fe II } \\
\text { weak He I (4471 ̊̊) }\end{array}$ & $\begin{array}{l}\mathrm{Mg} \text { triplet, suitable for the determination of } v \sin i \\
\text { of minor importance for fast rotators }\end{array}$ \\
\hline \multirow[t]{4}{*}{ Silicon } & $\mathrm{Si}$ II $4128 / 4130$ & weak Mn II/Fe II & \\
\hline & Si II 5041/5ß55 & weak Fe I & \\
\hline & Si II 6374 & two weak Mg II lines & \\
\hline & Si II 6371 & one weak Fe I line & \\
\hline \multirow[t]{2}{*}{ Calcium } & Ca II K 3934 & 31 weak blend lines & indicator for diffusion processes \\
\hline & & & $\mathrm{K}$ line used for detection of CS gas \\
\hline \multirow[t]{2}{*}{ Iron } & Fe II 4233 & weak Fe I & \\
\hline & Fe II 4584 & weak Ti II/Cr II & blend of four Fe II lines \\
\hline \multirow[t]{3}{*}{ Strontium } & Sr II 4077 & weak Fe I/Si II & diffusion indicator, weaker $\mathrm{Sr}$ II line with rising $T_{\text {eff }}$ \\
\hline & Sr II 4215 & weak $\mathrm{Cr}$ II/Fe I & diffusion indicator, weaker Sr II line with rising $T_{\text {eff }}$ \\
\hline & Sr II 4305 & weak Fe II/Ti II & diffusion indicator, weaker Sr II line with rising $T_{\text {eff }}$ \\
\hline Barium & Ba II 4554 & weak Fe II/Cr II/S II & diffusion indicator, weaker Ba II line with rising $T_{\text {eff }}$ \\
\hline
\end{tabular}

our calculations. The completed O I model atom was compared with the old version and a study by Przybilla et al. (2000) who have carried out a study on NLTE effects of oxygen and applied their model atom to the three stars, Vega (A0 V), $\eta$ Leo (A0 Ib), and HD 92207 (A0 Ie). The value for the oxygen abundance of the standard star Vega $\left(T_{\text {eff }}=9500 \mathrm{~K}, \log g=4.0,[\mathrm{M} / \mathrm{H}]=\right.$ -0.5 ) obtained with the O I model atom used in this work for the O I 7774 line is 8.52 which is accordance with 8.59 calculated by Przybilla et al. (2000). The Vega spectrum used for the abundance analysis of oxygen was recorded by I.K. \& M.H. in January 2000 with the $1.52-\mathrm{m}$ telescope of OHP equipped with the AURÉLIE spectrograph (Kamp et al. 2002).

For most of the program stars the NLTE corrections are in the range of -0.3 dex to -0.7 dex. Somewhat exceptional is the large correction for HR 3717 with -1.18 dex. The reason for this is the low gravity of $\log g=3.22$ - the second lowest of the whole sample. A lower surface gravity leads to a weaker coupling between the levels because the collisional rates decrease due to lower electron and gas pressures. Therefore NLTE effects are more important.

\subsection{Neon}

The neon model atom was developed by J. Graf $\left(2000^{2}\right)$ and consists of $45 \mathrm{Ne}$ I levels, $47 \mathrm{Ne}$ II levels, and 120 transitions. The atomic data was obtained from the Opacity Project (Seaton et al. 1992) and the NIST compilation. The oscillator strengths

\footnotetext{
${ }^{2}$ http://www.astrophysik. uni-kiel.de/d-pubalpha_ hol.html
}

are based on Seaton (1998) and Kurucz \& Peytremann (1975). The NLTE corrections are negative for all sample stars. While neon shows conspicuous overabundances in LTE for some stars, the NLTE abundances are rather solar. This is of importance for the question of weak stellar winds and will be discussed later.

\subsection{Magnesium}

The $\mathrm{MgI} / \mathrm{II}$ atom contains 99 levels and 71 line transitions and is an improved version by Gigas (1988) of the model developed by Lemke (1986). The model atom was adapted by Gigas for the use with ATLAS6 models having a frequency grid of 336 points. This grid defines the ODF averaged mean intensities and is used for the computation of the photoionization rates. For the calculations carried out in the course of this study the more recent ATLAS9 version was used and therefore the extended frequency grid containing 1212 points had to be implemented in the model atom. As outlined by Gigas (1988) the corrections for $\mathrm{Mg} 4481$ are expected to be negative. This result is confirmed here. Nevertheless the deviations from LTE are small, usually well below -0.2 dex. Therefore LTE is a good approximation of $\mathrm{Mg} 4481$ abundances for late B stars.

\subsection{Silicon}

The silicon atom used was developed by Wedemeyer (2001). It includes 115 energy levels of $\mathrm{Si}$ I/II and 84 lines. The NLTE calculations show negative corrections for the sample stars with the lowest temperatures whereas the deviations for 
Table 3. Energy levels of the O I model atom.

\begin{tabular}{|c|c|c|}
\hline No. & $E[\mathrm{eV}]$ & Designation \\
\hline 1 & 0.01 & $2 p^{43} \mathrm{P}$ \\
\hline 2 & 1.97 & $2 p^{41} D$ \\
\hline 3 & 4.19 & $2 \mathrm{p}^{4}{ }^{1} \mathrm{~S}$ \\
\hline 4 & 9.15 & $3 s^{5} S^{o}$ \\
\hline 5 & 9.52 & $3 s^{3} S^{o}$ \\
\hline 6 & 10.74 & $3 p^{5} \mathrm{P}$ \\
\hline 7 & 10.99 & $3 p^{3} \mathrm{P}$ \\
\hline 8 & 11.84 & $4 s^{5} S^{o}$ \\
\hline 9 & 11.93 & $4 s^{3} S^{o}$ \\
\hline 10 & 12.08 & $3 d^{5} D^{o}$ \\
\hline 11 & 12.09 & $3 d^{3} D^{o}$ \\
\hline 12 & 12.29 & $4 p^{5} \mathrm{P}$ \\
\hline 13 & 12.36 & $4 p^{3} \mathrm{P}$ \\
\hline 14 & 12.54 & $3 s^{3} D^{o}$ \\
\hline 15 & 12.66 & $5 s^{5} S^{o}$ \\
\hline 16 & 12.70 & $5 s^{3} S^{o}$ \\
\hline 17 & 12.73 & $3 s^{1} D^{o}$ \\
\hline 18 & 12.75 & $4 d^{5} D^{o}$ \\
\hline 19 & 12.76 & $4 d^{3} D^{o}$ \\
\hline 20 & 12.77 & $4 f^{5} \mathrm{~F}$ \\
\hline 21 & 12.77 & $4 \mathrm{f}^{3} \mathrm{~F}$ \\
\hline 22 & 12.85 & $5 p^{5} \mathrm{P}$ \\
\hline 23 & 12.88 & $5 p^{3} \mathrm{P}$ \\
\hline 24 & 13.02 & $6 s^{5} S^{o}$ \\
\hline 25 & 13.04 & $6 s^{3} S^{o}$ \\
\hline 26 & 13.07 & $5 d^{5} D^{o}$ \\
\hline 27 & 13.07 & $5 d^{3} D^{o}$ \\
\hline 28 & 13.07 & $5 \mathrm{f}^{5} \mathrm{~F}$ \\
\hline 29 & 13.07 & $5 f^{3} \mathrm{~F}$ \\
\hline 30 & 0.0 & CONT \\
\hline
\end{tabular}

the hotter ones turn to positive values. About $50 \%$ of the program stars show solar values within the error limits.

\subsection{Calcium}

The Ca I/II model was developed by W. Steenbock and is based on the work of Watanabe \& Steenbock (1985) on the Sun and Procyon. The improved version used for this study contains 125 levels with 100 transitions. The corrections for the Ca II K line are generally small and positive in most cases. Only for three stars do we find $|\Delta \log \epsilon| \geq 0.2$ dex. Most of the stars which have non-solar $\mathrm{Ca}$ abundances within the error limits show conspicuous NLTE underabundances up to -0.85 dex. This will be discussed further in Sect. 7 .

\subsection{Iron}

An iron model containing 99 energy levels of $\mathrm{FeI}$ and Fe II with 75 lines was developed by W. Steenbock \& T. Gehren. An extensive study using this model in a temperature range between $7000 \mathrm{~K}$ and $12000 \mathrm{~K}$ was carried out by
Table 4. Line transitions of the O I model atom: Lower level number (Low), upper level number (Up), wavelength and $\log g f$-value (Wiese et al. 1996).

\begin{tabular}{|c|c|c|c|c|c|c|c|}
\hline Low & Up & $\lambda[\AA]$ & $\log g f$ & Low & $\mathrm{Up}$ & $\lambda[\AA]$ & $\log g f$ \\
\hline 1 & 2 & 6333.79 & -9.652 & 7 & 16 & 7256.4 & -0.799 \\
\hline 1 & 3 & 2972.29 & -10.478 & 7 & 19 & 7004.1 & -0.410 \\
\hline 1 & 4 & 1355.60 & -5.238 & 7 & 25 & 6048.1 & -1.286 \\
\hline 1 & 5 & 1303.5 & -0.330 & 7 & 27 & 5960.2 & -1.140 \\
\hline 1 & 9 & 1040.1 & -1.084 & 8 & 12 & 27637.0 & 0.868 \\
\hline 1 & 11 & 1026.6 & -0.743 & 8 & 22 & 12267.0 & -1.100 \\
\hline 1 & 14 & 989.46 & -0.303 & 9 & 12 & 34850.8 & -4.248 \\
\hline 1 & 16 & 977.19 & -1.527 & 9 & 13 & 28927.0 & 0.666 \\
\hline 1 & 17 & 974.07 & -4.108 & 9 & 23 & 13077.0 & -1.180 \\
\hline 1 & 19 & 972.47 & -0.906 & 10 & 20 & 18021.0 & 1.401 \\
\hline 1 & 25 & 951.59 & -1.849 & 10 & 22 & 16110.0 & -1.628 \\
\hline 1 & 27 & 949.39 & -1.246 & 10 & 28 & 12511.0 & 0.606 \\
\hline 2 & 3 & 5577.34 & -8.930 & 11 & 13 & 45596.0 & 0.442 \\
\hline 2 & 5 & 1641.305 & -5.654 & 11 & 21 & 18244.0 & 1.188 \\
\hline 2 & 14 & 1172.504 & -4.512 & 11 & 23 & 15666.0 & -1.218 \\
\hline 2 & 17 & 1152.15 & -0.280 & 11 & 29 & 12570.0 & 0.380 \\
\hline 3 & 5 & 2325.452 & -7.950 & 12 & 15 & 33075.0 & 0.655 \\
\hline 4 & 6 & 7773.4 & 0.700 & 12 & 18 & 26507.0 & 1.231 \\
\hline 4 & 7 & 6728.16 & -4.398 & 12 & 19 & 26207.0 & -3.215 \\
\hline 4 & 12 & 3947.4 & -1.766 & 12 & 24 & 16872.0 & -0.371 \\
\hline 5 & 6 & 10167.3 & -4.553 & 12 & 26 & 15891.0 & 0.261 \\
\hline 5 & 7 & 8446.5 & 0.492 & 13 & 16 & 36607.0 & 0.463 \\
\hline 5 & 13 & 4368.2 & -1.709 & 13 & 18 & 31393.6 & -3.142 \\
\hline 5 & 23 & 3693.4 & -2.767 & 13 & 19 & 30977.0 & 1.037 \\
\hline 6 & 8 & 11299.0 & 0.407 & 13 & 25 & 18229.0 & -0.594 \\
\hline 6 & 10 & 9263.9 & 1.156 & 13 & 27 & 17453.0 & -0.092 \\
\hline 6 & 11 & 9207.46 & -3.831 & 14 & 21 & 54557.28 & -2.260 \\
\hline 6 & 15 & 6455.0 & -0.589 & 14 & 23 & 36666.0 & -1.328 \\
\hline 6 & 18 & 6157.3 & 0.034 & 14 & 29 & 23259.0 & -1.131 \\
\hline 6 & 19 & 6142.28 & -4.420 & 18 & 20 & 973302.0 & -2.467 \\
\hline 6 & 24 & 5437.7 & -1.067 & 18 & 22 & 131395.0 & -0.136 \\
\hline 6 & 26 & 5331.5 & -0.540 & 18 & 28 & 38808.0 & 1.326 \\
\hline 7 & 9 & 13165.0 & 0.222 & 19 & 23 & 103975.7 & -0.229 \\
\hline 7 & 10 & 11374.0 & -3.861 & 19 & 29 & 39463.0 & 1.126 \\
\hline 7 & 11 & 11299.0 & 0.407 & 20 & 26 & 33135.0 & -0.288 \\
\hline 7 & 14 & 7992.0 & -2.094 & & & & \\
\hline
\end{tabular}

Rentzsch-Holm (1996). Iron shows only slightly negative abundance corrections below -0.1 dex in NLTE. Therefore LTE is a good approximation for the program stars. This is in accordance with the results of Rentzsch-Holm (1996).

\subsection{Strontium}

The strontium model atom (Belyakova et al. 1997) incorporates 41 levels of $\mathrm{Sr}$ II including the ground state of $\mathrm{Sr}$ III. The level energies were taken from Moore (1952) and Lindgard \& Nielsen (1977), and the $f$ values were adopted from Wiese \& Martin (1980), Lindgard \& Nielsen (1977), and Kurucz (1994). Further details concerning the model atom can be found in Belyakova et al. (1997). Non-LTE calculations for strontium were kindly performed by Elena Belyakova using the Kazan non-LTE code (Belyakova et al. 1999). With one exception (HR 2948) strontium lines of sufficient strength were only found in sample stars below $12000 \mathrm{~K}$. Strontium is overabundant in LTE in most of those objects. The NLTE corrections are positive in all cases and quite conspicuous. This deserves some comments in Sect. 7.

\subsection{Barium}

The Ba II model by D. Gigas (1988) includes 42 levels and 36 transitions. Only in one star was a barium line of sufficient strength found. A new ATLAS9 frequency grid had to be 
Table 5. LTE and NLTE abundances. The NLTE corrections and abundances of C, O and Ne are given with respect to the solar values.

\begin{tabular}{|c|c|c|c|c|c|c|c|c|c|c|}
\hline \multirow[t]{2}{*}{ HR } & {$[\mathrm{He} / \mathrm{H}]$} & {$[\mathrm{C} / \mathrm{H}]$} & $\Delta \log \epsilon$ & {$[\mathrm{C} / \mathrm{H}]$} & {$[\mathrm{O} / \mathrm{H}]$} & $\Delta \log \epsilon$ & {$[\mathrm{O} / \mathrm{H}]$} & {$[\mathrm{Ne} / \mathrm{H}]$} & $\Delta \log \epsilon$ & {$[\mathrm{Ne} / \mathrm{H}]$} \\
\hline & & LTE & & NLTE & LTE & & NLTE & LTE & & NLTE \\
\hline 806 & 0.44 & - & - & - & 0.8 & -0.46 & 0.34 & - & - & - \\
\hline 1070 & 0.08 & -0.15 & 0.15 & 0.00 & 0.9 & -0.55 & 0.35 & - & - & - \\
\hline 1092 & 1.05 & 0.36 & 0.09 & 0.45 & 1.3 & -0.74 & 0.56 & - & - & - \\
\hline 1214 & 0.19 & -0.17 & 0.22 & 0.05 & 0.8 & -0.58 & 0.22 & 0.15 & -0.15 & 0.00 \\
\hline 1339 & - & -0.08 & 0.09 & 0.01 & 0.9 & -0.55 & 0.35 & - & - & - \\
\hline 1582 & 0.38 & -0.05 & 0.24 & 0.19 & 1.3 & -0.86 & 0.44 & 0.3 & -0.28 & 0.02 \\
\hline 1723 & 0.30 & - & - & - & 0.9 & -0.35 & 0.55 & - & - & - \\
\hline 1728 & - & - & - & - & 0.0 & -0.33 & -0.33 & - & - & - \\
\hline 1973 & 0.01 & - & - & - & -1.5 & -0.43 & -1.93 & - & - & - \\
\hline 2056 & 0.17 & -0.17 & 0.2 & 0.03 & 1.0 & -0.64 & 0.36 & 0.1 & -0.17 & -0.07 \\
\hline 2948 & -0.25 & 0.45 & -0.05 & 0.40 & 0.0 & -0.35 & -0.35 & 0.45 & -0.17 & 0.28 \\
\hline 3158 & - & - & - & - & 0.5 & -0.31 & 0.19 & - & - & - \\
\hline 3439 & 0.42 & - & - & - & -1.5 & -0.33 & -1.83 & - & - & - \\
\hline 3717 & 0.60 & -0.16 & -0.03 & -0.19 & 1.5 & -1.18 & 0.32 & 0.58 & -0.47 & 0.11 \\
\hline 4116 & - & - & - & - & 0.8 & -0.38 & 0.42 & - & - & - \\
\hline 4119 & 0.29 & -0.23 & 0.15 & -0.08 & 1.0 & -0.62 & 0.38 & 0.45 & -0.19 & 0.26 \\
\hline 4943 & - & - & - & - & 1.0 & -0.38 & 0.62 & - & - & - \\
\hline 5501 & - & - & - & - & 0.8 & -0.42 & 0.38 & - & - & - \\
\hline 5685 & - & - & - & - & 1.6 & -0.7 & 0.9 & - & - & - \\
\hline 5994 & - & - & - & - & 0.8 & -0.44 & 0.36 & - & - & - \\
\hline 6628 & 0.12 & -0.2 & 0.24 & 0.04 & 1.3 & -0.7 & 0.6 & 0.15 & -0.15 & 0.00 \\
\hline 6633 & - & - & - & - & 1.16 & -0.53 & 0.63 & - & - & - \\
\hline 6647 & -0.71 & -0.42 & 0.14 & -0.28 & 0.5 & -0.61 & -0.11 & 0.55 & -0.41 & 0.14 \\
\hline 6668 & - & - & - & - & 1.1 & -0.47 & 0.63 & - & - & - \\
\hline 6878 & - & - & - & - & -0.5 & -0.35 & -0.85 & - & - & - \\
\hline 7337 & 0.41 & -0.14 & 0.15 & 0.01 & 1.0 & -0.51 & 0.49 & 0.0 & -0.08 & -0.08 \\
\hline 8781 & - & - & - & - & 1.2 & -0.4 & 0.8 & - & - & - \\
\hline
\end{tabular}

provided for the model atom. The NLTE correction of 0.4 dex is in accordance with the findings of Gigas (1988).

\subsection{Helium}

For helium no NLTE calculations were carried out in the course of this study. Leone \& Lanzafame (1998) investigated the behaviour of several He I lines - including two lines at $5876 \AA$ and $6678 \AA$ which were as well examined in this work in a wide temperature range. Their comparison between their NLTE calculations and LTE models of various authors reveal that for spectral types between A0 and B3 equivalent widths and thus LTE and NLTE abundances of these two lines are in concordance. Therefore in the case of the scrutinized He I lines LTE is a good approximation for the program stars.

\section{Narrow absorptions in Ca II K}

In some cases we found narrow absorption features in the Ca II K line and - after removing telluric lines - in $\mathrm{NaD}$ of two stars as well (see Table 8). They are similar to those detected in our former work on A stars (Holweger et al. 1999). The most prominent star with narrow $\mathrm{Ca}$ II $\mathrm{K}$ absorptions is $\beta$ Pictoris. Its Ca II K profiles show redshifted narrow absorption features with a time dependency of the order of weeks and months which is attributed to the infall of cometarylike objects which evaporate as they approach the stellar surface (see e.g. Lagrange-Henri et al. 1992). In addition about $30 \%$ of the 28 normal A stars and $18 \lambda$ Bootis stars studied by Holweger et al. (1999) show detectable Ca II K features. The question arises as to whether these absorptions are of interstellar or rather of circumstellar origin. As outlined in Holweger et al. (1999) such features have only been found in stars with $v \sin i>80 \mathrm{~km} \mathrm{~s}^{-1}$. This is true for the B stars discussed in this work as well. Therefore a stellar property is correlated with the occurrence of Ca II K feature which supports a circumstellar origin. Holweger \& Rentzsch-Holm (1995) give the following tentative interpretation: for stars with circumstellar gas concentrated in a disk-like structure the column density of absorbing gas along the line of sight will be at its maximum if the disk is viewed edge-on. Therefore circumstellar absorption lines should be detected preferably in objects with 
Table 6. LTE and NLTE abundances. The NLTE corrections and abundances of Mg, Si and Ca are given with respect to the solar values.

\begin{tabular}{|c|c|c|c|c|c|c|c|c|c|}
\hline HR & $\begin{array}{r}{[\mathrm{Mg} / \mathrm{H}]} \\
\mathrm{LTE}\end{array}$ & $\Delta \log \epsilon$ & $\begin{array}{r}{[\mathrm{Mg} / \mathrm{H}]} \\
\text { NLTE }\end{array}$ & $\begin{array}{r}{[\mathrm{Si} / \mathrm{H}]} \\
\mathrm{LTE}\end{array}$ & $\Delta \log \epsilon$ & $\begin{array}{l}\mathrm{Si} / \mathrm{H}] \\
\mathrm{NLTE}\end{array}$ & $\begin{array}{r}{[\mathrm{Ca} / \mathrm{H}]} \\
\mathrm{LTE}\end{array}$ & $\Delta \log \epsilon$ & $\begin{array}{r}{[\mathrm{Ca} / \mathrm{H}]} \\
\mathrm{NLTE}\end{array}$ \\
\hline 806 & -0.09 & -0.04 & -0.13 & 0.02 & -0.11 & -0.09 & -0.04 & 0.14 & 0.10 \\
\hline 1070 & -0.46 & -0.1 & -0.56 & -0.26 & -0.14 & -0.40 & -0.52 & 0.2 & -0.32 \\
\hline 1092 & -0.08 & -0.09 & -0.17 & 0.2 & -0.18 & 0.02 & 0.14 & 0.18 & 0.32 \\
\hline 1214 & -0.26 & -0.11 & -0.37 & -0.18 & 0.27 & 0.09 & -0.45 & 0.03 & -0.42 \\
\hline 1339 & -0.4 & -0.06 & -0.46 & 0.04 & -0.18 & -0.14 & 0.16 & 0.15 & 0.31 \\
\hline 1582 & 0.09 & -0.07 & 0.02 & -0.04 & 0.45 & 0.41 & -0.57 & -0.05 & -0.62 \\
\hline 1723 & 0.18 & -0.04 & 0.14 & 0.02 & -0.15 & -0.13 & -0.14 & 0.17 & 0.03 \\
\hline 1728 & -1.2 & -0.03 & -1.23 & -0.37 & -0.09 & -0.46 & -0.98 & 0.13 & -0.85 \\
\hline 1973 & -0.52 & -0.1 & -0.62 & -0.25 & -0.10 & -0.35 & -0.69 & 0.17 & -0.52 \\
\hline 2056 & 0.31 & -0.06 & 0.25 & -0.03 & 0.36 & 0.33 & 0.01 & -0.02 & -0.01 \\
\hline 2948 & -0.72 & -0.04 & -0.76 & -0.33 & 0.33 & 0.00 & -0.08 & -0.06 & -0.14 \\
\hline 3158 & -0.8 & -0.05 & -0.85 & -0.25 & -0.12 & -0.37 & -0.68 & 0.13 & -0.55 \\
\hline 3439 & -0.09 & -0.05 & -0.14 & -0.21 & -0.15 & -0.36 & -0.82 & 0.18 & -0.64 \\
\hline 3717 & -0.19 & 0.07 & -0.12 & -0.48 & 0.41 & -0.07 & 0.98 & 0.11 & 1.09 \\
\hline 4116 & -0.5 & -0.05 & -0.55 & 0.06 & -0.18 & -0.12 & -0.32 & 0.17 & -0.15 \\
\hline 4119 & 0.00 & -0.06 & -0.06 & 0.02 & 0.29 & 0.31 & 0.52 & 0.09 & 0.61 \\
\hline 4943 & -0.5 & -0.04 & -0.54 & -0.02 & -0.12 & -0.14 & -0.4 & 0.17 & -0.23 \\
\hline 5501 & -0.37 & -0.07 & -0.44 & -0.09 & -0.14 & -0.23 & -0.24 & 0.15 & -0.09 \\
\hline 5685 & -1.3 & -0.02 & -1.32 & 1.0 & -0.20 & 0.80 & 0.0 & 0.21 & 0.21 \\
\hline 5994 & -0.44 & -0.05 & -0.49 & -0.07 & -0.12 & -0.19 & -0.24 & 0.09 & -0.15 \\
\hline 6628 & -0.02 & -0.05 & -0.07 & -0.02 & -0.24 & -0.26 & 0.09 & 0.21 & 0.30 \\
\hline 6633 & 0.07 & -0.07 & 0.00 & 0.12 & -0.24 & -0.12 & -0.17 & 0.17 & 0.00 \\
\hline 6647 & -0.36 & -0.17 & -0.53 & -0.11 & 0.54 & 0.43 & -0.19 & -0.19 & -0.38 \\
\hline 6668 & 0.06 & -0.07 & -0.01 & 0.12 & -0.25 & -0.13 & -0.53 & 0.17 & -0.36 \\
\hline 6878 & -0.69 & -0.08 & -0.77 & -0.26 & -0.16 & -0.42 & -0.46 & 0.12 & -0.34 \\
\hline 7337 & -0.24 & -0.06 & -0.30 & -0.04 & -0.21 & -0.25 & -0.62 & 0.17 & -0.45 \\
\hline 8781 & -0.1 & -0.07 & -0.17 & -0.13 & -0.29 & -0.42 & -0.1 & 0.16 & 0.06 \\
\hline
\end{tabular}

$\sin i \approx 1$. Hence the chance to find a star with narrow absorption lines and low $v \sin i$ is small.

\section{Discussion}

The results presented in Tables 5-7 reveal that the abundances of the program stars are widely distributed. A closer inspection of the data shows some interesting correlations.

\subsection{Diffusion}

In some stars cool enough to show detectable strontium and barium lines, we detect NLTE overabundances of these elements. Most obviously, the diffusion indicators barium and strontium are clearly overabundant in HR 1728, HR 1973, HR 2948, HR 3439, HR 5501, HR 5994, HR 6633, HR 6668, and HR 6878. Furthermore, some of these stars (HR 1973 and HR 3439 as well as HR 6633 and HR 6668) show similar abundance patterns which suggests that they can be ascribed to the same physical process. In the widely accepted diffusion scenario (Michaud 1970; Michaud \& Charland 1986) this indicates element separation by diffusion in their outer layers. In addition to that the three other scrutinized diffusion indicators (oxygen, magnesium, and calcium) are deficient in the strontium and barium overabundant stars HR 1728, HR 1973, HR 3439, and HR 6878. This strengthens the diffusion hypothesis. Some program stars show abundance anomalies of the diffusion indicators to a lesser extent while other stars, namely
HR 1723, HR 2056 and HR 4119, have abundances which are close to the solar values. For stars with deviations from solar composition similar abundance patterns were as well found for HR 3158, HR 4116, HR 4943 and HR 8781 and for HR 806, HR 1070, HR 1092 and HR 1723. We illustrate these correlations in Fig. 2. All stars where both strontium and calcium were analysed are overabundant in strontium and solar or underabundant in calcium. In fact, only one of these stars, namely HR 6633, has a solar value of calcium. The values obtained for strontium reveal as well a correlation with the oxygen abundances: while strontium is radiatively driven outwards oxygen sinks down. For strontium and magnesium the situation is similar: all stars where magnesium is deficient show overabundances of the diffusion indicator strontium. In contrast, neon - which is not an indicator of diffusion - shows a very small scatter and illustrates the quality of the NLTE-analysis.

The anomalies detected in the program stars do not occur to the same extent in all stars but show star-to-star variations. This suggests that a counterpart to diffusion may blur the abundance anomalies. B-type stars are fast rotators. The rotation of a star causes accelerations which lead to the mixing of the stellar envelope. The ability of stellar rotation to inhibit effective diffusion processes was already mentioned in the fundamental paper by Michaud (1970). This so-called meridional mixing is wellknown to occur in A stars. The effectivity of the mixing of the stellar atmosphere increases with increasing rotational velocity. 
Table 7. LTE and NLTE abundances. The NLTE corrections and abundances of $\mathrm{Fe}, \mathrm{Sr}$ and $\mathrm{Ba}$ are given with respect to the solar values.

\begin{tabular}{rrrrrrrrrr}
\hline \hline $\mathrm{HR}$ & {$[\mathrm{Fe} / \mathrm{H}]$} & $\Delta \log \epsilon$ & {$[\mathrm{Fe} / \mathrm{H}]$} & {$[\mathrm{Sr} / \mathrm{H}]$} & $\Delta \log \epsilon$ & {$[\mathrm{Sr} / \mathrm{H}]$} & {$[\mathrm{Ba} / \mathrm{H}]$} & $\Delta \log \epsilon$ & {$[\mathrm{Ba} / \mathrm{H}]$} \\
& $\mathrm{LTE}$ & & NLTE & LTE & & NLTE & LTE & & NLTE \\
\hline 806 & -0.01 & -0.02 & -0.03 & - & - & - & - & - & - \\
1070 & -0.41 & -0.02 & -0.43 & - & - & - & - & - & - \\
1092 & 0.29 & -0.08 & 0.21 & - & - & - & - & - & - \\
1214 & 0.07 & -0.05 & 0.02 & - & - & - & - & - & - \\
1339 & -0.42 & -0.01 & -0.43 & - & - & - & - & - & - \\
1582 & 0.04 & -0.05 & -0.01 & - & - & - & - & - & - \\
1723 & 0.09 & -0.03 & 0.06 & - & - & - & - & - & - \\
1728 & -0.44 & 0.00 & -0.44 & 0.02 & 0.49 & 0.51 & - & - & - \\
1973 & 0.54 & -0.03 & 0.51 & 0.65 & 0.60 & 1.25 & 0.9 & 0.4 & 1.3 \\
2056 & -0.06 & -0.04 & -0.10 & - & - & - & - & - & - \\
2948 & 0.14 & -0.04 & 0.10 & 2.26 & 0.26 & 2.52 & - & - & - \\
3158 & -0.51 & -0.01 & -0.52 & - & - & - & - & - & - \\
3439 & 1.39 & -0.01 & 1.38 & 2.38 & 0.40 & 2.78 & - & - & - \\
3717 & -0.21 & -0.06 & -0.27 & - & - & - & - & - & - \\
4116 & -0.51 & -0.01 & -0.52 & - & - & - & - & - & - \\
4119 & 0.19 & -0.05 & 0.14 & - & - & - & - & - & - \\
4943 & -0.21 & -0.02 & -0.23 & - & - & - & - & - & - \\
5501 & -0.26 & -0.02 & -0.28 & -0.30 & 0.65 & 0.35 & - & - & - \\
5685 & - & - & - & - & - & - & - & - & - \\
5994 & 0.24 & -0.02 & 0.22 & 0.60 & 0.55 & 1.15 & - & - & - \\
6628 & -0.26 & -0.06 & -0.32 & - & - & - & - & - & - \\
6633 & 0.34 & -0.04 & 0.30 & 0.18 & 0.77 & 0.59 & - & - & - \\
6647 & 0.14 & -0.05 & 0.09 & - & - & - & - & - & - \\
6668 & 0.29 & -0.03 & 0.26 & -0.07 & 0.77 & 0.70 & - & - & - \\
6878 & 0.14 & -0.03 & 0.11 & 0.40 & 0.60 & 1.00 & - & - & - \\
7337 & -0.31 & -0.04 & -0.35 & - & - & - & - & - & - \\
8781 & -0.06 & -0.04 & -0.10 & - & - & - & - & - & - \\
\hline & & & & & & & & &
\end{tabular}

Table 8. Equivalent widths of the detected narrow absorption features.

\begin{tabular}{|c|c|c|}
\hline \multirow[t]{2}{*}{ HR } & \multicolumn{2}{|c|}{ Equivalent width [mÅ] } \\
\hline & Ca II K & $\mathrm{NaD}_{1,2}$ \\
\hline 1070 & 9 & $-/-$ \\
\hline 1092 & 121 & $300 / 241$ \\
\hline 1582 & 40 & $30 / 16$ \\
\hline 2056 & 13 & $-/-$ \\
\hline 4943 & 32 & $-/-$ \\
\hline
\end{tabular}

Therefore in a certain sample of stars with abundance anomalies, we expect to find more pronounced deviations from the normal values in slow rotators than in fast rotating stars. This is nicely illustrated for the case of $\lambda$ Bootis stars in Holweger $\&$ Rentzsch-Holm (1995). Their Fig. 13 shows the calcium abundances of their $\lambda$ Bootis stars investigated as a function of rotational velocity. While stars with low rotation show conspicuous underabundances, the fast rotators approach solar values.

Figure 3 shows the abundances of calcium as a function of $v \sin i$. Abundances of stars with rotational velocities below $100 \mathrm{~km} \mathrm{~s}^{-1}$ show a large scatter while the scatter for fast rotators $\left(v \sin i>100 \mathrm{~km} \mathrm{~s}^{-1}\right)$ is less pronounced. The abundances of the two fastest rotators (HR 1092 and HR 5685) are afflicted with larger errors of 0.4 dex. Therefore the result obtained from Fig. 3 is in accordance with simulations carried out by Turcotte \& Charbonneau (1993) which have shown that rotational velocities above $125 \mathrm{~km} \mathrm{~s}^{-1}$ lead to an effective mixing of the stellar envelope.

Given our presented results we suggest that the variety of diffusion-driven abundance patterns known from A stars continues to the temperature regime of late B stars and conclude that the abundance anomalies discovered in some of the program stars can be attributed to diffusion combined with meridional mixing.

\subsection{Weak stellar winds}

In OB stars radiatively driven stellar winds are common (Abbott 1980, 1982; Friend \& Abbott 1986). It is well-known that these winds not only lead to substantial mass loss with rates up to $\sim 10^{-4} M_{\odot} /$ year (Maeder 1983) but also affect the chemical composition of stars above $20 M_{\odot}$ (Kilian 1992).

The masses of early-type B stars are far above the masses of the program stars but the objects investigated in this study are situated in a transition region between the diffusion dominated stellar surfaces and the wind driven atmospheres of hot B stars. In this temperature range the occurrence of weak stellar winds with a mass-loss rate of $10^{-14}-10^{-12} M_{\odot} /$ year is theoretically predicted (Babel 1995). These winds may compete with diffusion processes and are expected to affect the chemical composition of the stellar surface. Furthermore, these winds play an important role in the widely accepted diffusion scenario: the abundance patterns of several stars where diffusion 

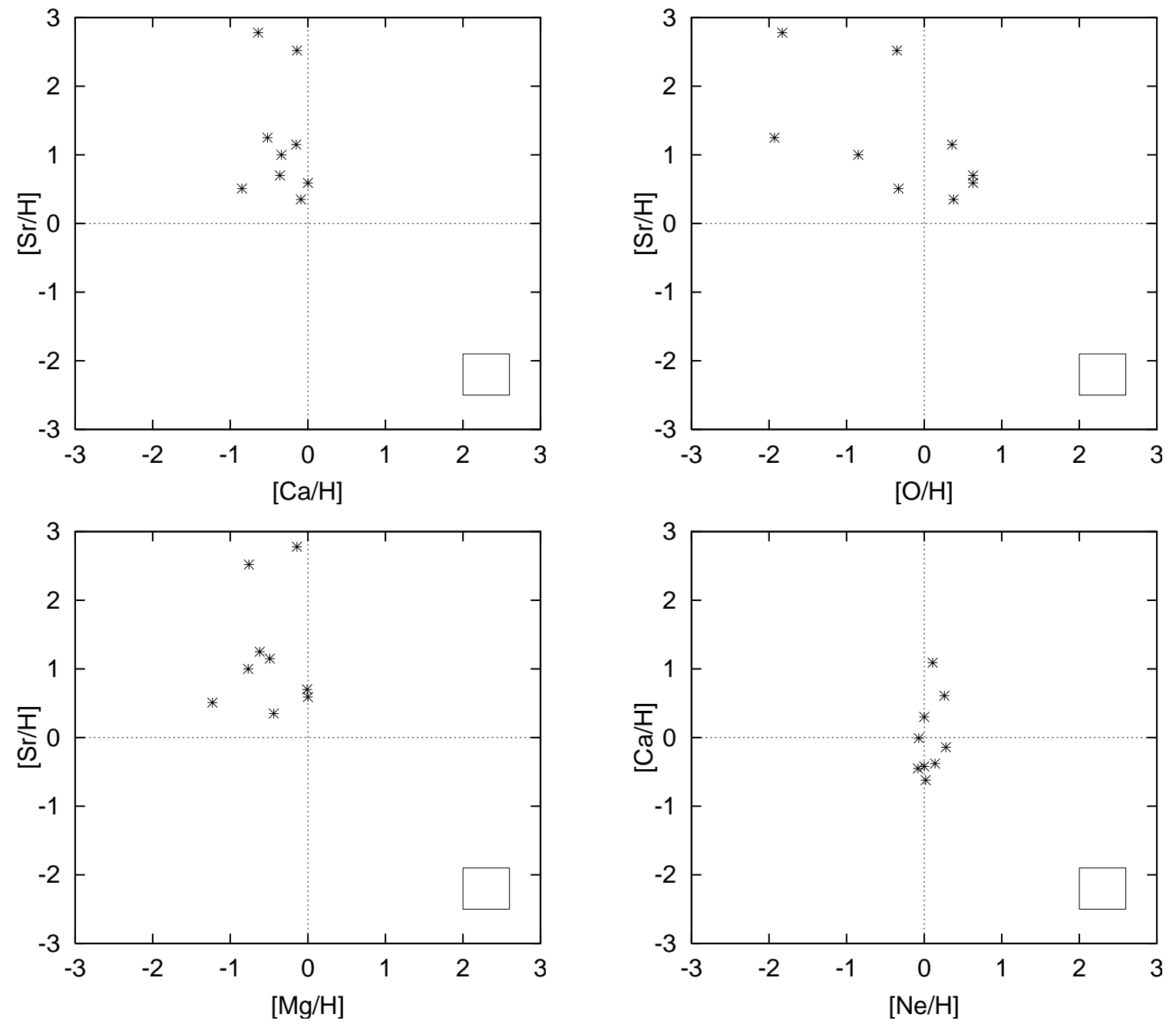

Fig. 2. Correlations between NLTE abundances of various elements. In the lower right corner of the plots there is an error box representing the typical error of $\pm 0.3 \mathrm{dex}$ of our abundance analysis. Note the small scatter of neon abundances which is in sharp contrast to the scatter of the diffusion indicator calcium.

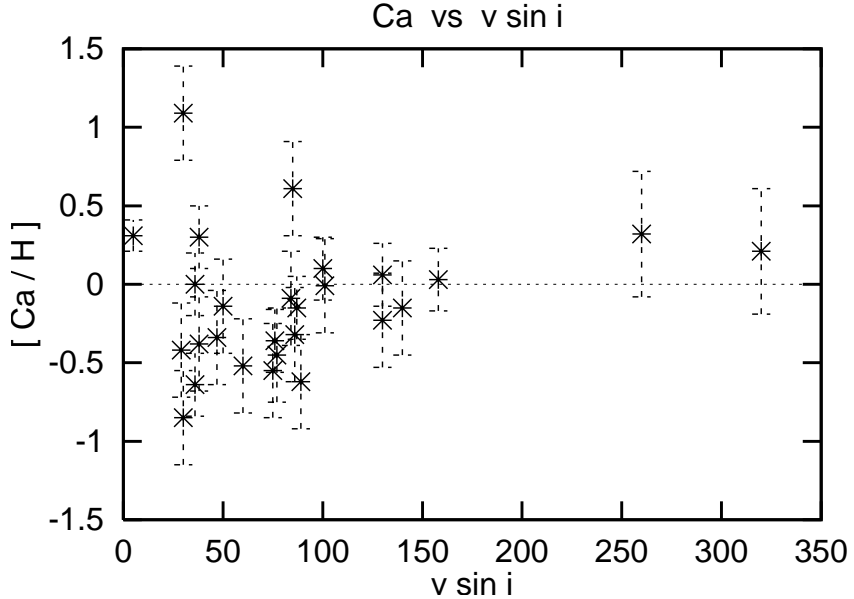

Fig. 3. The NLTE abundances of calcium as a function of $v \sin i$.

is efficient adopt in most cases the existence of a weak stellar wind to explain mild inconsistencies between theory and observation (Alecian 1996; Hui-Bon Hoa \& Alecian 1998).
A stellar wind of the order of $10^{-14}-10^{-12} M_{\odot} /$ year would be mild enough not to inhibit diffusion. Unfortunately, in contrast to the massive stellar winds of OB stars there is no spectroscopic evidence for the existence of weak stellar winds to date. A theoretical attempt to detect weak stellar winds in the temperature range of late B stars was proposed by Landstreet et al. (1998). The basic idea is that abundant elements like carbon, oxygen, and neon which are not expected to be affected by radiative acceleration may accumulate in the outer layers of a mass-losing star and thus serve as trace elements for the detection of weak stellar winds. The temperature range examined by Landstreet et al. (1998) is $8000 \leq T_{\text {eff }} \leq 15000 \mathrm{~K}$. In view of these weak stellar winds investigations of two Ne I lines at $6402 \AA$ and $6506 \AA$ were carried out in the course of this study. Unfortunately neon is only observable for stars with $T_{\text {eff }}>11500 \mathrm{~K}$. Nevertheless, the LTE results (Table 5) reveal overabundances of $\mathrm{Ne}$ I of up to $0.58 \mathrm{dex}$ in at least five program stars. This would have been the first spectroscopic detection of weak stellar winds. Since Ne I is known to be affected by large NLTE effects, this result is suspect. In order to examine this in more detail NLTE effects had to be taken into account. 
The neon model atom developed by Graf (2000) reveals that the abundance corrections for neon are negative for all investigated stars. This leads to essentially solar neon abundances of all stars within the error limits (Table 5). Therefore weak stellar winds were not detected in the course of this study which suggests that if suchlike winds are present their intensity is below $10^{-14} M_{\odot} /$ year.

This is in accordance with the results obtained for oxygen which is a tracer of these winds in cooler stars (Landstreet et al. 1998). Moreover, this confirms the findings of Dworetsky \& Budaj (2000) who analysed neon in a sample of normal B stars and HgMn stars. Their results reveal essentially solar neon abundances for normal B stars. For the only star in common with Dworetsky \& Budaj (2000) - HR 1339 - we considered the neon lines in the spectra as too weak to allow a reliable abundance determination. In a recent study (Budaj \& Dworetsky 2002) on radiative accelerations of late B stars they find the radiative accelerations well below the gravitational accelerations which leads them to predict underabundances for neon. As can be seen from Fig. 2 and Table 5 we do not find significant underabundances of neon for our stellar sample. This may be due to some competetive process, e.g. meridional mixing (see Sect. 7.1 and Fig. 3).

Nevertheless, the result achieved for the neon abundances demonstrates the importance of NLTE calculations in the investigated temperature range since an LTE analysis pretends the presence of weak stellar winds.

\section{Galactic abundance gradients}

Because of their brightness and as B stars are in general young objects they are often used for Galactic abundance studies (see e.g. Kaufer et al. 1994). The study of the chemical composition of stars and the variation of stellar abundances within the Galaxy is of fundamental importance for the understanding of Galactic evolution. The stars studied in this work cannot be used for that kind of investigations since they represent only a volume-limited sample within the solar neighbourhood. In order to obtain high $\mathrm{S} / \mathrm{N}$ spectra the program stars were taken from the Bright Star Catalogue (Hoffleit \& Warren 1991), therefore the stars lie in the solar vicinity (see Table 1). A different point is important here: the results of this work reveal that diffusion processes can occur in the outer layers of late B stars. As a consequence, the composition of such a star does not reflect the primordial abundances of the interstellar cloud it originated from and may therefore not be a reliable tracer for Galactic abundance studies if the effects of diffusion are neglected. This result is strengthened by the study of Luck et al. (2000) and reveals the importance of the investigation of late B stars.

\section{Conclusions}

The main results of this work can be summarized as follows: (1) A large fraction of late B stars show anomalous rather than solar abundances. (2) Combined with meridional mixing diffusion can explain the observed abundance patterns. (3) The result of the search for the occurrence of weak stellar winds of $\sim 10^{-14} M_{\odot} /$ year by using NLTE abundances as a diagnostic tool was negative. Thus mass loss rates must be below this limit. (4) Element separation processes seem to be common in late B stars. This constitutes a serious problem for using such stars for galactic abundance studies. (5) In five stars narrow absorptions in $\mathrm{Ca}$ II $\mathrm{K}$ have been found. They are rather of circumstellar than of interstellar origin.

Acknowledgements. We would like to express our gratitude to Dr. A. Kaufer, Dr. G. A. Galazutdinov, and Dr. F. A. Musaev for kindly providing the spectra. We thank E. Belyakova for performing the NonLTE calculations for Sr. We are grateful to the referee for helpful comments. This work was supported by DFG grants Ho 596/38-1 and Ho 596/38-2 and by the DLR under grant DLR 50 OR 0005.

\section{References}

Abbott, D. C. 1980, ApJ, 242, 1183

Abbott, D. C. 1982, ApJ, 259, 282

Anders, E., \& Grevesse, N. 1989, Geochim. Cosmochim. Acta, 53, 197

Friend, D. B., \& Abbott, D. C. 1986, ApJ, 311, 701

Adelman, S. J., \& Philip, A. G. D. 1996, MNRAS, 282, 1181

Alecian, G. 1996, A\&A, 310, 872

Allen, C. W. 1973, Astrophysical Quantities, 3rd ed. (London: Athlone Press)

Auer, L. H., \& Mihalas, D. 1973, ApJ, 184, 151

Babel, J. 1995, A\&A, 301, 823

Baschek, B., Scholz, M., \& Sedlmayr, E. 1977, A\&A, 207, 112

Belyakova, E. V., Mashonkina, L. I., \& Sakhibullin, N. A. 1999, A Rep, 43, 819

Belyakova, E. V., \& Mashonkina, L. I. 1997, A Rep, 41, 530

Budaj, J., \& Dworetsky, M. M. 2002, MNRAS, 337, 1340

Dworetsky, M. M., \& Budaj, J. 2000, MNRAS, 318, 1264

ESA, 1997, The Hipparcos and Tycho Catalogues, ESA SP-1200

Fitzpatrick, E. L., \& Massa, D. 1999, ApJ, 525, 1011

Gigas, D. 1986, A\&A, 165, 170

Gigas, D. 1988, A\&A, 192, 264

Gonzalez, J.-F., LeBlanc, F., Artu, M.-C., \& Michaud, G. 1995, A\&A, 297,223

Graf, J 2000, Diploma Thesis, Kiel

Hauck, B., \& Mermilliod, M. 1990, A\&AS, 86, 107

Hoffleit, D. E., \& Warren, W. H. jr. 1991, The Bright Star Catalogue, 5th rev. ed., Yale Univ. Obs., New Haven (BSC)

Holweger, H., Gigas, D., \& Steffen, M. 1986, A\&A, 155, 58

Holweger, H., Hempel, M., \& Kamp, I. 1999, A\&A, 350, 603

Holweger, H., Kock, M., \& Bard, A. 1995, A\&A, 296, 233

Holweger, H., \& Rentzsch-Holm, I. 1995, A\&A, 303, 819

Hui-Bon Hoa, A., \& Alecian, G. 1998, A\&A, 332, 224

Kamp, I., Hempel, M., \& Holweger, H. 2002, A\&A, 388, 978 (KHH)

Kaufer, A., Szeifert, Th., Krenzin, R, Baschek, B., \& Wolf, B. 1994, A\&A 289, 740

Kilian, J. 1992, A\&A, 262, 171

Krege, C. 1995, Diploma Thesis, Kiel

Kudritzki, R.-P., \& Hummer, D. G. 1990, ARA\&A, 28, 303

Kurucz, R. L. 1993, in Peculiar Versus Normal Phenomena in AType and Related Stars, ed. M. M. Dworetsky, F. Castelli, \& R. Faraggiana, ASP Conf. Ser., 44, 87

Kurucz, CD-ROMs 20-22, July 3, 1994 edition

Kurucz, R. L., \& Peytremann, E. 1975, A Table of Semiempirical $g f$ values, SAO Washington

Lagrange-Henri, A. M., Gosset, E., Beust, H., Ferlet, R., \& Vidal-Madjar, A. 1992, A\&A, 264, 637 
Landstreet, J. D., Dolez, N., \& Vauclair, S. 1998, A\&A, 333, 977

Lemke, M. 1986, Diploma Thesis, Kiel

Lemke, M. 1989, A\&A, 225, 125

Lemke, M. 1990, A\&A, 240, 331

Lemke, M. 1991, Internal Report, Department of Astronomy, University of Texas at Austin

Leone, F., \& Lanzafame, A. C. 1998, A\&A, 330, 306

Lindgard, A, \& Nielsen, S. E. 1977, Atomic Data Nucl. Data Tables, 19

Luck, R. E., Andrievsky, S. M., Kovtyukh, V. V., Korotin, S. A., \& Beletsky, Yu. V. 2000, A\&A, 361, 189

Maeder, A. 1983, A\&A 120, 113

Michaud, G. 1970, ApJ, 160, 641

Michaud, G., \& Charland, Y. 1986, ApJ, 311, 326

Moore, C. E. 1952, Atomic Energy Levels, Circ. NBS, 467

Napiwotzki, R., Schönberner, D., \& Wenske, V. 1993, A\&A, 268, 653

Paunzen, E., Kamp, I., Iliev, I. Kh., et al. 1999, A\&A, 345, 597

Piskunov, N. E., Kupka, F., Ryabchikova, T. A., Weiss, W. W., \& Jeffery, C. S. 1995, A\&AS, 112, 525

Przybilla, N., Butler, K., Becker, S. R., Kudritzki, R. P., \& Venn, K. A. 2000, A\&A, 359, 1085

Rentzsch-Holm, I. 1996, A\&A, 312, 966

Seaton, M. J. 1998, MNRAS, 300, L1

Seaton, M. J., Zeippen, C. J., Tully, J. A., et al. 1992, Rev. Mex. Astron. Astrofis., 23, 19
Smith, K. C. 1993, A\&A, 276, 393

Smith, K. C. 1996, A\&A, 305, 902

Smith, K. C., \& Dworetsky, M. M. 1993, A\&A, 274, 335

Steenbock, W., \& Holweger, H. 1984, A\&A, 130, 319

Stürenburg, S. 1993, A\&A, 277, 139

Stürenburg, S., \& Holweger, H. 1990, A\&A, 237, 125

Takeda, Y. 1992, PASJ, 44, 309

Tayal, S. S. 1992, J. Phys. B: At. Mol. Opt. Phys., 25, 2639

Tayal, S. S., \& Henry, R. J. W. 1989, Phys. Rev. A, 39, 4531 van Thiel, T. 1997, Diploma Thesis, Kiel

Turcotte, S., \& Charbonneau P. 1993, ApJ, 413, 376

Van Regemorter, H. 1962, ApJ, 136, 906

Venn, K. A., \& Lambert, D. L. 1990, ApJ, 363, 234

Wang, S., \& Mc Conkey, J. W. 1992, J. Phys. B: A. Mol. Opt. Phys., 25,5461

Watanabe, T., \& Steenbock, W. 1985, A\&A, 149, 21

Wedemeyer, S. 2001, A\&A, 373, 998

Wiese, W. L., \& Martin, G. A. 1980, Wavelengths and Transition Probabilities for Atoms and Atomic Ions, part II, NSRDS-NBS 68, Washington D.C.

Wiese, W. L., Fuhr, J. R., \& Deters, T. M. 1996, J. Phys. Chem. Ref. Data Monograph No. 7

Wohler, S. 1996, Diploma Thesis, Kiel

Wolff, S. C. 1983, The A Stars: Problems and Perspectives, NASA SP-463 